\title{
EXPERIMENTAL AND NUMERICAL STUDY OF THE DEVELOPMENT OF SWIRLING FLOW AND FLAME DYNAMICS AND COMBUSTION CHARACTERISTICS AT BIOMASS THERMO-CHEMICAL CONVERSION
}

\author{
Inesa Barmina ${ }^{1}$, Raimonds Valdmanis ${ }^{1}$, Harijs Kalis ${ }^{2}$, Maksims Marinaki ${ }^{2}$ \\ ${ }^{1}$ Institute of Physics, University of Latvia; \\ ${ }^{2}$ Institute of Mathematics and Computer Science, University of Latvia \\ barmina@sal.lv,harijs.kalis@lu.lv
}

\begin{abstract}
The focus of this study is to investigate the main factors determining the development of swirling flow dynamics and to correlate the development of the non-premixed swirling flame characteristics at biomass thermo-chemical conversion with the evolution of the confined swirling flow velocity fields in a pilot device which combines a biomass gasifier and a combustor. This study includes complex experimental study and numerical modelling of the development of velocity fields for confined non-reacting swirling flows and flame, as well as the development of swirling flame velocity fields and combustion characteristics at biomass thermochemical conversion under effects of various inlet conditions, such as the inlet nozzle diameter at the bottom of the combustor, primary and swirling air supply rates in the device. The results show that the development of the swirling flow velocity field first of all is closely related to the inlet nozzle diameter, which for the fixed primary and secondary air supply rates strongly affects the upstream and downstream swirling airflow formation and swirl intensity, which are highly responsible for the mixing of combustible volatiles with the axial air flow, for the ignition and combustion of volatiles. The results also show that the development of the swirling flow velocity field depends on the air supply rate which affects the development of the combustion dynamics and composition of emission through the variation of the downstream flow structure and the air excess ratio in the flame reaction zone.
\end{abstract}

Keywords: swirling flows, biomass pellets, combustion dynamics, mathematical model.

\section{Introduction}

The application of swirling flows for the design of combustion systems is important due to the swirl-induced formation of a toroidal recirculation zone, which results in enhanced mixing of a fuel with the air, in stabilization of combustion dynamics and in greater combustion efficiency. The comprehensive research of the confined swirling flows includes an experimental study and a numerical analysis of the development of the downstream flow structure and combustion dynamics by varying the flow geometry, boundary conditions and swirl intensity [1-5]. These studies have shown that relatively small variations of the inlet conditions and configuration of the swirl combustor can result in unpredictable variations of the flow patterns and main flame characteristics. Moreover, the complex research of the swirling non-reacting flow and flame dynamics in a cylindrical channel has revealed that the development of swirling flow patterns and flame structure correlate with the formation of downstream and upstream swirling air flows which are responsible for biomass gasification, mixing of the axial flow of volatiles with the air, ignition and combustion of volatiles and for the formation of main combustion characteristics [6]. Further research has shown that the formation of the downstream and upstream swirling flow patterns and swirling flame structure is highly sensitive to the variations of inlet conditions, i.e. the inlet nozzle geometry as well as the air supply rate and the mode in the device. To assess the influence of the inlet conditions on the development of upstream and downstream swirling flow patterns, flow structure and main combustion characteristics, the present study includes complex experimental study and numerical modelling of the development of the velocity field for confined non-reacting swirling flows and flame by varying the diameter of the inlet nozzle, which is located at the bottom of the combustor, as well as of the primary and secondary air supply rates into the device. The correlation between the development of the flow patterns and the main combustion characteristics is observed.

\section{Experimental details}

The experimental device combines a cylindrical batch-size biomass gasifier (1) and a combustor (2) of diameter $60 \mathrm{~mm}$ and device total length up to $500 \mathrm{~mm}$ [6]. The experiments were carried out under the conditions when the biomass gasifier and the combustor were separated by an inlet nozzle, which is located at the base of the combustor (3) (Fig. 1). 


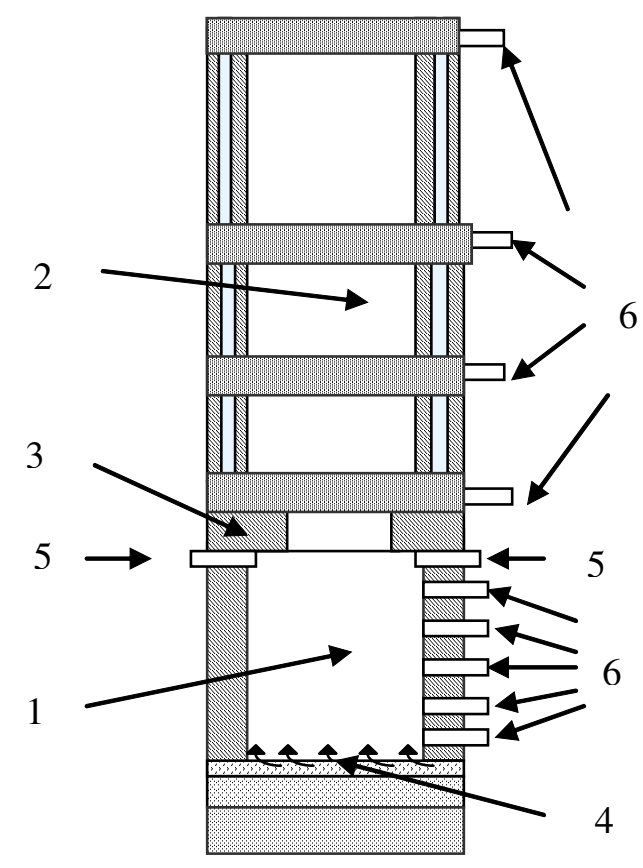

Fig. 1. Sketch of the experimental device: 1 - biomass gasifier; 2 - combustor water-cooled sections; 3 - annular inlet nozzle of the combustor; 4 - primary axial air supply; 5 - secondary swirling air supply; 6 - orifices for diagnostic tools

To assess the influence of inlet conditions on the development of cold non-reacting swirling flow dynamics, the inlet nozzles with diameters of $20 \mathrm{~mm}, 40 \mathrm{~mm}$ and $60 \mathrm{~mm}$ were used. The primary air was supplied axially at the bottom of the gasifier (4) by varying the air supply rate from 0 to $301 \cdot \mathrm{min}^{-1}$. The secondary swirling air was supplied from two tangential air nozzles, which were attached to the channel walls and located close below the inlet nozzle of the combustor (5). In recent study, the secondary air supply could be varied from $301 \cdot \mathrm{min}^{-1}$ to $901 \cdot \mathrm{min}^{-1}$. The development of upstream and downstream swirling flow dynamics and the formation of flow structure were studied providing the measurements of the radial and axial velocity profiles for upstream and downstream flows. The measurements of velocity profiles for cold non-reacting flows were made using a thermoprobe and online data registration with a Testo 350 gas analyzer. The effect of inlet conditions on the development of swirling flame dynamics and on the formation of flame temperature and composition profiles was studied by local measurements of the flow velocity, flame temperature and composition. Local measurements of the flame temperature were made by Pt/Pt-Rh thermocouples with data online registration by a Pico logger. Local measurements of the flame composition, i.e. the mass fraction of volatiles $\left(\mathrm{CO}, \mathrm{H}_{2}\right)$, the volume fraction of the main product $\left(\mathrm{CO}_{2}\right)$, as well as the air excess ratio $(\alpha)$ and the combustion efficiency, were made using a gas sampling probe and a gas analyzer Testo 350 . Calorimetric measurements of the cooling water flow were used to assess the impact of inlet conditions on the heat output of the device and on the average values of the produced heat energy at different stages of biomass thermo-chemical conversion.

\section{Experimental results}

The development of the cold non-reacting flow velocity profiles for fixed primary and secondary air supply rates $\left(10 \mathrm{l} \cdot \mathrm{min}^{-1}\right.$ and $\left.60 \mathrm{l} \cdot \mathrm{min}^{-1}\right)$ and fixed inlet nozzle diameter $(d=40 \mathrm{~mm})$ is illustrated in Fig. 2 (a-c). The measurements of the flow axial and tangential velocity profiles confirm the formation of a downstream swirling airflow in the combustor $(L / D>0)$ and an upstream swirling airflow in the gasifier $(L / D<0)$. The upstream swirling airflow propagates up to the bottom of the gasifier $(L / D \approx-2)$ and then reverses from the gasifier base producing a very complicated flow pattern. As follows from Fig. 2-a,b, by increasing the distance from the inlet nozzle the tangential velocity $(w)$ of the upstream swirling airflow tends to decrease and the swirl motion of the upstream airflow gradually approaches the flow centreline. The most pronounced decrease of the tangential velocity is observed close to the flow centreline $(r / R<0.5)$, where the formation of the reversed downstream axial flow 
occurs. This correlation between the decrease of the upstream swirling flow velocity and the increase of the downstream axial flow velocity confirms that the swirl flow reversing from the base of the gasifier results in a decrease of the swirl momentum of the upstream flow along with the enhanced formation of the downstream axial flow which gradually decays as the flow propagates downstream (Fig. 2-a). The formation of the upstream swirling airflow and reversed downstream axial airflow results in local variations of the upstream flow swirl number $\left(S \approx 2 / 3 \cdot u_{\varphi a v} / u_{z a v}\right)$ and swirl intensity (Fig. 2-c). As follows from Fig. 2-c, the upstream flow swirl number reaches the maximum value at the interface between the reversed axial downstream flow and the tangential upstream flows $(0.3<r / R<0.5)$ indicating that the axial downstream flow is surrounded by the upstream air swirl.
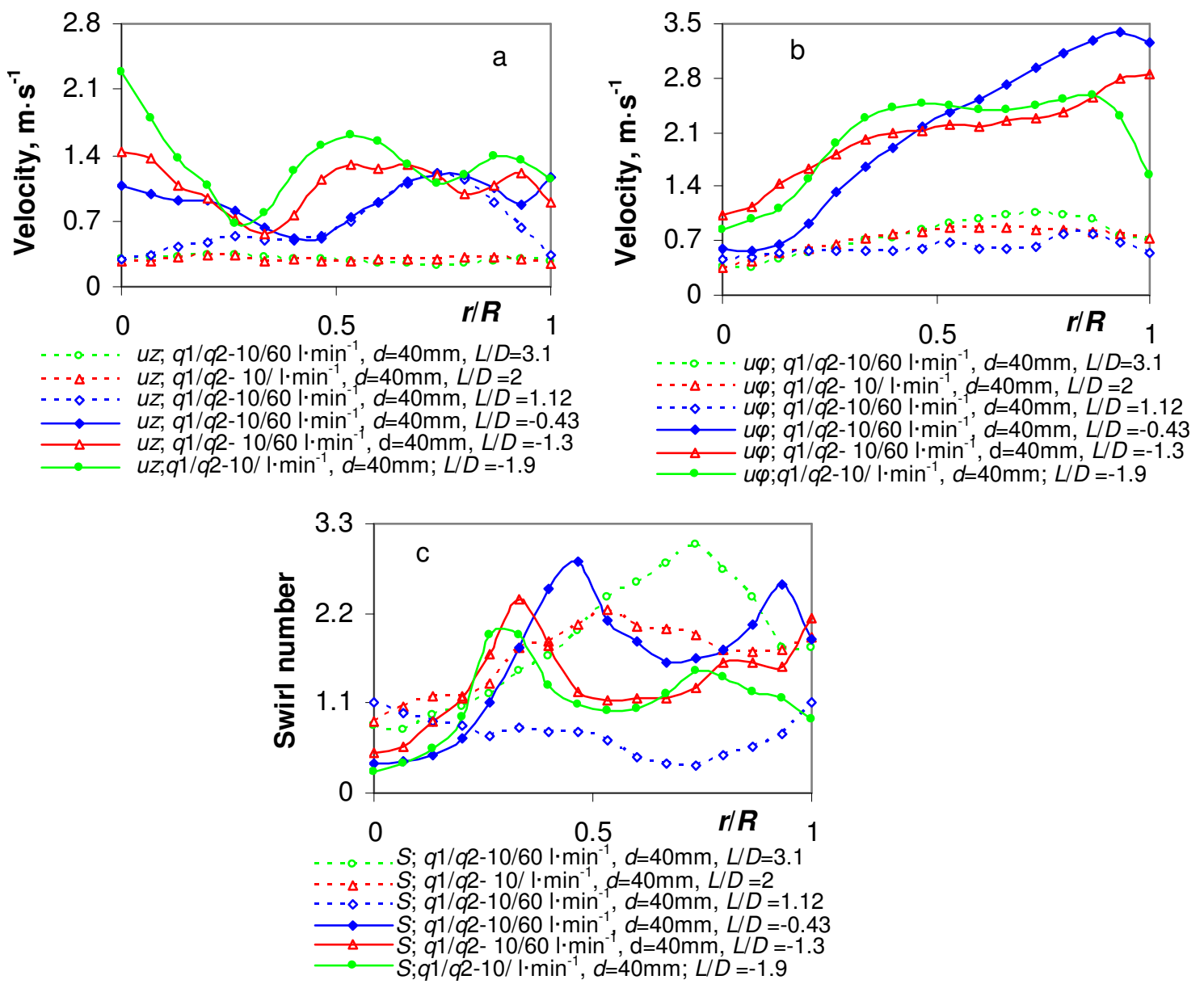

Fig. 2. Formation of radial profiles of downstream $(L / D>0)$ and upstream non-reacting swirling airflow $(\boldsymbol{L} / \boldsymbol{D}<\mathbf{0})$ : a - radial profiles of axial velocity; b - radial profiles of tangential velocity; $\mathrm{c}$ - radial profiles of swirl number (3)

The development of the radial and axial velocity profiles of the downstream flow above the inlet nozzle $(L / D>0)$ differs from that of the upstream flow. By increasing the distance from the inlet nozzle, the downstream swirling airflow, due to the action of the centrifugal force, gradually expands with the decrease of the local and average values of the axial $\left(u_{a v}\right)$ airflow velocity (Fig. 2, 3-a), whereas the tangential velocity of the downstream flow (Fig. 3-b) slightly increases, with the correlating increase of the swirl intensity. Moreover, due to the radial downstream flow expansion, the peak values of the tangential velocity and swirl intensity shift towards the channel walls - in a reverse direction, as it is observed for the upstream flow (Fig. 2-c). Finally, it should be emphasized that the development of the non-reacting flow dynamics is highly influenced by the variations of the swirling air supply rates and by the variations of the inlet nozzle opening. The results of the experimental study have shown that both the axial and the tangential flow velocity of the downstream and upstream flows can be increased by increasing the secondary swirling air supply rate (Fig. 3-a) and decreasing the inlet nozzle diameter (Fig. 3-b). 

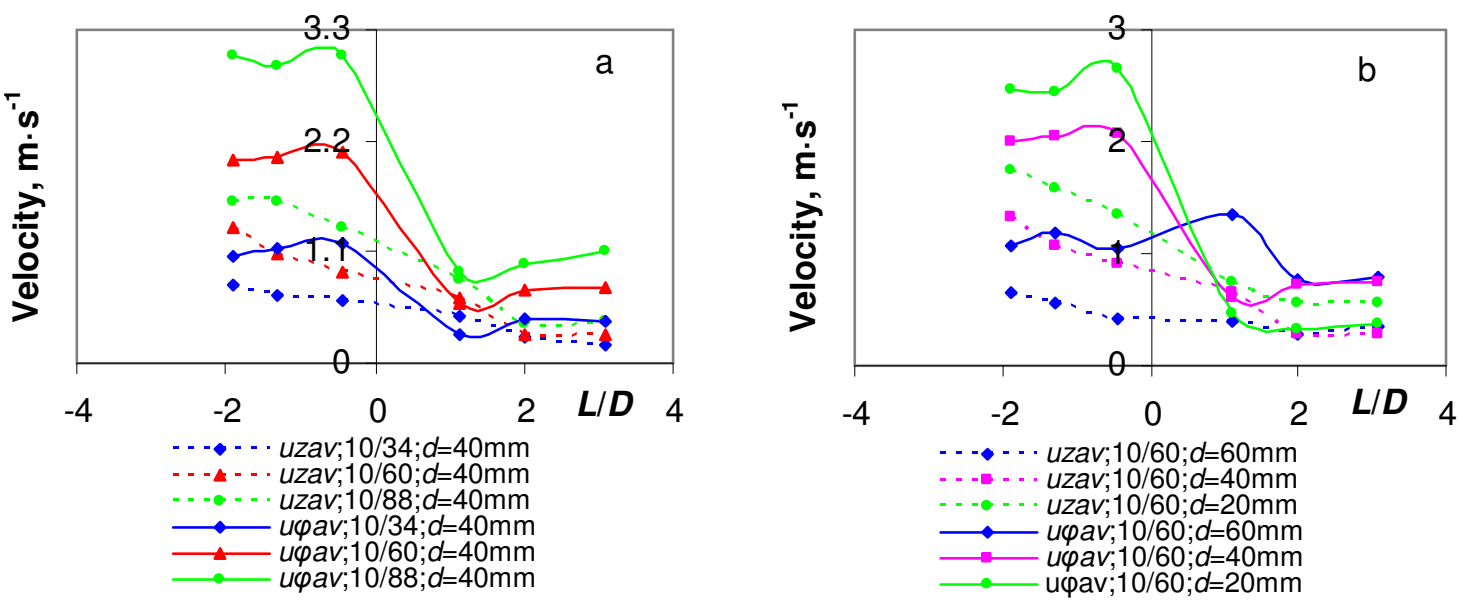

Fig. 3. Formation of axial profiles of the non-reacting swirling airflows: a - development of axial velocity average values; $b$ - development of tangential velocity average values

The enhanced upstream swirling flow formation with reflection from the biomass layer also follows from the experimental study of the swirling flame flow field formation at biomass gasification and combustion of volatiles (Fig. 4-a,b). By analogy with the formation of upstream flow patterns for cold non-reacting flows, the upstream swirling flow velocity and swirl intensity rapidly decrease towards the flow centerline $(r / R<0.5)$, promoting so the correlating increase of the axial velocity of the reflected (reversed) downstream flow. Moreover, by increasing the secondary swirling air supply the reflected airflow demonstrates also a slight increase of the tangential velocity and swirl intensity close to the flame centerline $(r / R<0.1)$ (Fig. 4-b) with direct influence of the downstream flow patterns.
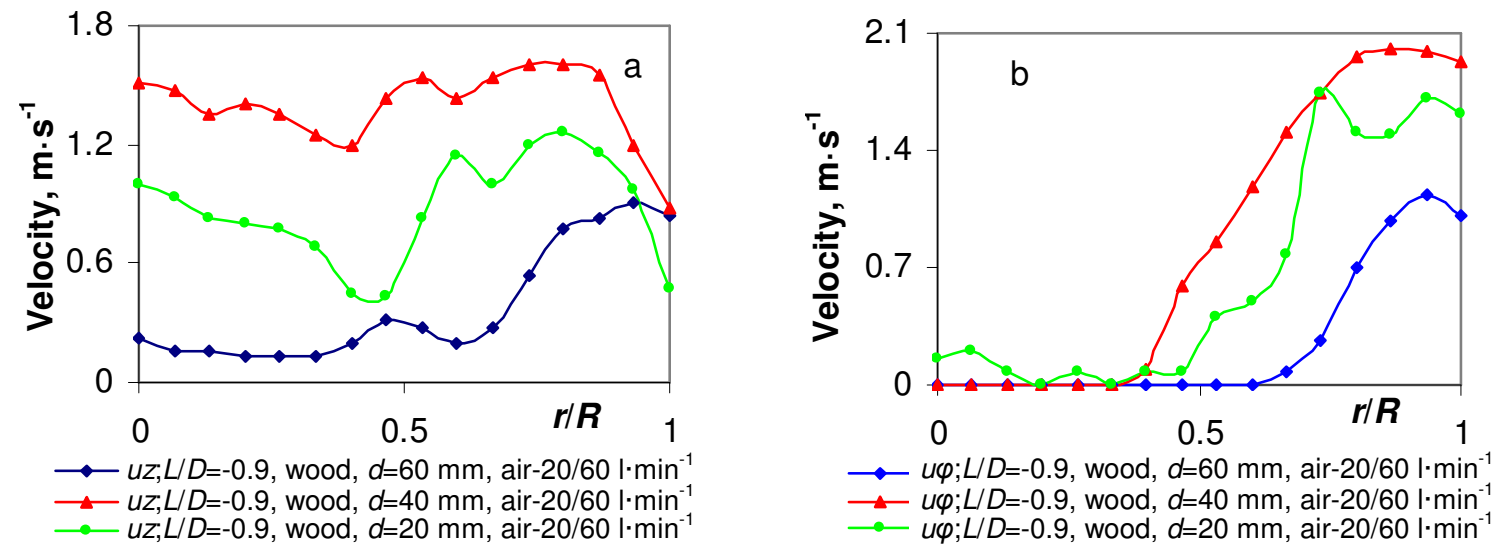

Fig. 4. Formation of radial profiles of the swirling flame upstream flow: a - radial profiles of the upstream axial velocity; $b$ - radial profiles of the upstream tangential velocity

The upstream swirling airflow formation and its propagation to the surface of the biomass layer, which is loaded into the gasifier, is highly responsible for primary mixing of the axial flow of volatiles with the air swirl, which occurs close to the surface of the biomass layer and results in an increase of the mass fraction of combustible volatiles $\left(\mathrm{CO}, \mathrm{H}_{2}\right)$ in the reflected (reversed) axial downstream flow (Fig. 5-a). This is confirmed by a kinetic study of the flame composition at different stages of the swirling flame flow formation. The pronounced increase of the mass fraction of volatiles in the products is observed at the primary volatile pre-combustion stage $(t<500 \mathrm{~s})$ with the correlating decrease of the volatiles mass fraction and free oxygen volume fraction in the products during the successive stage of volatiles flaming combustion $(t \approx 500-1200 \mathrm{~s})$ (Fig. 5-a, b). 

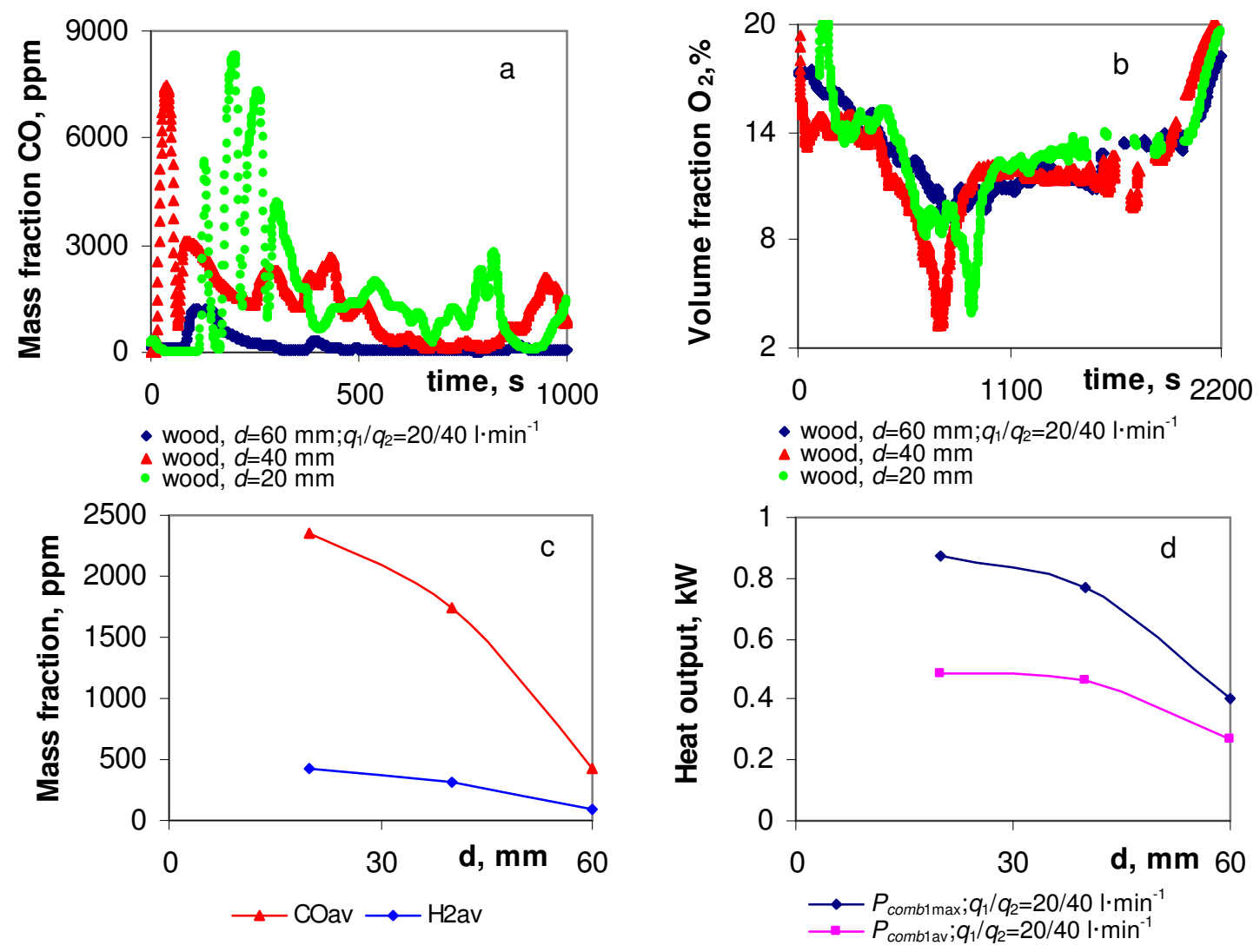

Fig. 5. The inlet nozzle diameter versus the main combustion characteristics: $a, b$ - kinetics of the swirling flame flow composition; $\mathrm{c}$-average values of the mass fraction of volatiles in the products at the volatile pre-combustion stage; $d$ - heat output of the device during the flaming combustion of volatiles

By analogy with the cold non-reacting flows, the swirling flame flow patterns are affected by the formation of upstream and reflected (reversed) downstream flows, the velocity of which depends on the inlet nozzle diameter, i.e. decreasing the inlet nozzle diameter results in the enhanced formation of upstream swirling and downstream axial flows in the device (Fig. 3-b). The effect of the inlet nozzle diameter on the formation of flow patterns and mixing of flame components is confirmed by the measurements of the average values of the mass fraction of volatiles in the products for different inlet nozzle diameters. These measurements reveal that decreasing the inlet nozzle diameter with the enhanced upstream swirling flow formation results in an enhanced mixing of volatiles with the air swirl and in an enhanced mass transfer of volatiles into the flame reaction zone by increasing the average values of the mass fraction of volatiles in the products during the pre-combustion of the volatiles (Fig. 5-c). With the air excess ratio in the reaction zone $\alpha>1.5-2$, the enhanced mass transfer of volatiles into the combustor results in improvement of the combustion characteristics decreasing so the air-to-fuel ratio and the air excess ratio in the flame reaction zone to $\alpha \approx 1-1.5$ with the correlating increase of the heat output from the device (Fig. 5-d) during the flaming combustion of the volatiles.

For mathematical modelling of 2D axially symmetric, compressible, swirling flow (velocities $u_{r}$, $u_{z}, u_{\varphi}$ ) in a cylindrical pipe-combustor (Fig. 6) with the radius $r_{0}=0.05 \mathrm{~m}$, length $z=z_{0}=0.1 \mathrm{~m}$, primary axial air and oxidant supply $r \in\left[0, r_{1}\right]$ (velocities $U_{0}=0.01 \mathrm{~m} \cdot \mathrm{s}^{-1}$ ), secondary swirlingazimuthal air supply $r \in\left[r_{1}, r_{0}\right]$ (velocities $V_{0}>U_{0}$ ), with the simple chemical reaction (mass-fraction of the reactant $\mathrm{C}$, temperature $\mathrm{T}$ ), the process is described by four Euler and two reaction-diffusion dimensionless equations in the cylindrical coordinates $(r, z)$ at a time $t$ [8].

The equations were made dimensionless minding the parameters $r, x=z / r_{0},\left(x_{0}=z_{0} / r_{0}\right), \rho$, $u=u_{r} / U_{0}, w=u_{z} / U_{0}$, by scaling the lengths to $r_{0}$, at the inlet the density to $\rho_{0}=1 \mathrm{~kg} \cdot \mathrm{m}^{-3}$, the velocities to $U_{0}$, the circulation $v=u_{\varphi} \cdot r$ to $V_{0} \cdot r_{0}$, the temperature to $T_{0}=300 \mathrm{~K}$ (the swirl number $S=V_{0} / U_{0}$ ). For the dimensionless pressure $\mathrm{p}$, we consider a perfect gas model: $p=\rho T$. 
We use the hydrodynamic stream function in the following way: $r \rho w=\partial \Psi / \partial r$, $r \rho u=-\partial \Psi / \partial x$.

At the inlet $x=0$, the following boundary conditions (BC) are applied: $u=0, T=1$ for $r \in[0,1]$, and $w=1, \mathrm{C}=1, \Psi=0.5 r^{2}, v=0$ for $r \in\left[0, r_{1}\right] ; \Psi=q, v=4 r \frac{\left(r-r_{1}\right)(1-r)}{\left(1-r_{1}\right)^{2}}, w=0, C=0$ at $r>r_{1}$ ( $q=r_{1}^{2} / 2$ is the dimensionless fluid volume), $r_{1}=0.5,0.75$. At the inlet, we have a uniform jet flow at $r<r_{1}$ and rotation at $r>r_{1}$ with the maximum azimuthal velocity equal to unity. Other BC can be found in [8].

The distribution of the stream function, of the components of velocity and temperature versus the swirl number and varying flow geometry $\left(r_{1}\right)$ was calculated using the implicit finite difference scheme and the ADI method. In Fig. 6, the distribution of the stream function for $r_{1}=0.5, S=3$ is presented; the inlet flow is deflected towards the wall forming a prominent vortex that blocks the central part of the pipe.

The results of the numerical simulation for different values on $\mathrm{S}$ and $\mathrm{r}_{1}$ are listed in Table 1, where $\min \Psi$ is the minimum value of the stream function (without vortices $0<\Psi<q$ ). One can see the occurrence of the vortex breakdown at a swirl number around $S \geq 0.9$ in the recirculation zone. The breakdown zone moves upstream and increases in size with the increase of the swirl number. The development of vortices depends on the diameter of the secondary air supply through the inlet.

Table 1

\section{Minimum values of the stream function $(\min \Psi)$ for different swirl numbers $S$ and $r_{1}$}

\begin{tabular}{|c|c|c|c|c|}
\hline $\boldsymbol{S}$ & $\boldsymbol{r}_{\mathbf{1}}$ & $\boldsymbol{\operatorname { m i n }} \boldsymbol{\Psi}$ & $\boldsymbol{r}_{\mathbf{1}}$ & $\min \boldsymbol{\Psi}$ \\
\hline 3.5 & 0.5 & -0.0913 & 0.75 & -0.0235 \\
\hline 2.0 & 0.5 & -0.0386 & 0.75 & -0.0161 \\
\hline 0.9 & 0.5 & -0.0061 & 0.75 & -0.0035 \\
\hline
\end{tabular}
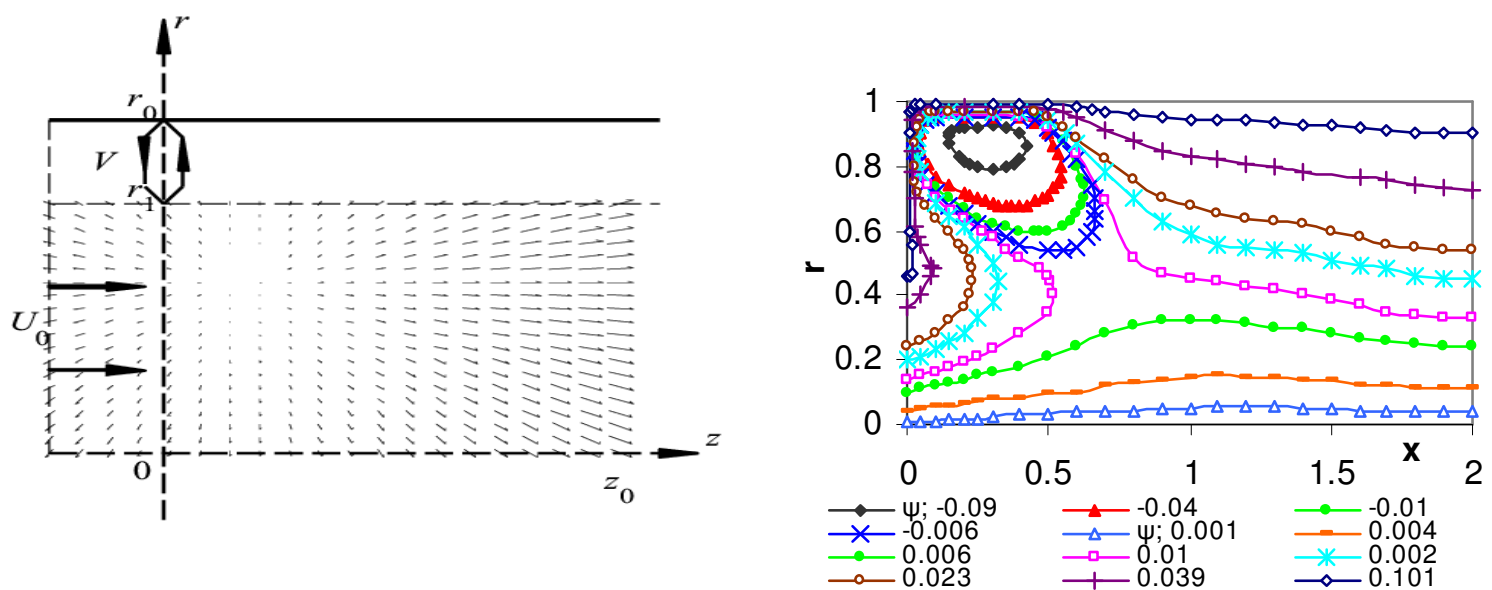

Fig. 6. Sketch of the mathematical model (left) and the distribution of the stream functions $\Psi$ (right) for $r_{1}=0.5, S=3$

\section{Conclusions}

From the results presented above the following conclusions can be drawn.

1. For the given system configuration, the development of non-reacting swirling flow dynamics in a cylindrical channel and flow patterns is influenced by the formation of upstream swirling and downstream axial flows, the velocity of which depends on the primary and secondary air supply rates and on the inlet nozzle diameter of the combustor. The enhanced upstream flow formation can be obtained by reducing the diameter of the inlet nozzle and by increasing the secondary air supply into the device.

2. The enhanced upstream swirling flow formation at biomass thermal decomposition boosts the improved mixing of the axial flow of volatiles with the air swirl, whereas the swirl flow reversing 
and the axial downstream flow formation evolve the enhanced mass transport of volatiles into the combustor by varying the air-to-fuel ratio in the flame reaction zone and by improving the combustion conditions at the flaming stage of volatiles, the development of which depends on the diameter of the inlet nozzle and on the secondary air supply into the device.

\section{Acknowledgements}

The authors would like to express their gratitude for the financial support from the Latvian Council of Science in the framework of Latvian Research Cooperation Project No. 623/2014.

\section{References}

1. Syred N., Beer J.M. Combustion in swirling flows: A review. Combustion and Flame, vol. 23, 1974, pp. 143-201.

2. Candel S., Durox D., Schuller T., Palies P. Bourgouin J.F., Moeck J.P. Progress and challenges in swirling flame dynamics. Comptes Rendus Mecanique, vol. 340, 2012, pp. 758-768.

3. Sweeney M.S., Hochgreb S., Dunn M.J., Barlow R.S. The structure of turbulent stratified and premixed methane/air flames II: Swirling flows. Combustion and Flame, vol. 159, 2012, pp. 2912-2929.

4. Naskar M., Roy D., Majumder S. Numerical analysis and control of the recirculation bubble strength of turbulent confined jet flow using inlet swirl. International Journal of Engineering, Science and Technology, vol.2, 2010, pp. 1-16.

5. Yang Y., Kær S.K., Yin C. Numerical study and validation of one swirling flame, Proceedings of the European Combustion Meeting, 2011.

6. Abricka M., Barmina I., Valdmanis R., Zake M. Experimental and numerical study of swirling flow dynamics. Latvian Journal of Physics and Technical Sciences, vol. 51, 2014, pp. 25-40.

7. Abricka M., Barmina I., Valdmanis R., Zake M., Kalis H. Experimental and numerical studies on integrated gasification and combustion of biomass. Chemical Engineering Transactions, vol. 50, 2016, p.127-132.

8. Kalis H., Barmina I., Zake M., Koliskins A. Mathematical modelling and experimental study of electrodynamic control of swirling flame flows. Proc. Int. Conf. "Engineering for Rural Development”, Jelgava, 25-27.05.2016, 134-141. 